\title{
Risa y Risoterapia en el campo de la salud y sus beneficios en algunos contextos: Una breve revisión
}

\section{Laughter and Laughter therapy in the field of health and its benefits in some contexts: A brief review}

\author{
Rodríguez Quezada, Fanny ${ }^{1 *}$ y Rojas Verdugo, Erica Paola ${ }^{2}$ \\ ${ }^{1}$ Universidad Católica de Cuenca \\ ${ }^{2}$ Universidad Católica de Cuenca, Sede Cañar \\ *frodriguezq@ucacue.edu.ec
}

\begin{abstract}
Resumen
La risa como una expresión de bienestar, alegría y descarga emocional, ha sido considerada una terapia alternativa y no invasiva dentro del ámbito de la salud. El objetivo del presente trabajo es determinar los beneficios de la risa en el campo de la medicina, sus características, la risoterapia como técnica fundamentada en la risa y la utilidad de ésta en ámbitos como la salud cardiovascular, el estrés y el cáncer a través de una breve revisión de su literatura.
\end{abstract}

Palabras clave: Risa, Risoterapia, Salud.

\begin{abstract}
Laughter as an expression of well-being, joy and emotional discharge, has been considered an alternative and non-invasive therapy within the field of health. The objective of the present study is to determine the benefits of laughter in the field of medicine, its characteristics, laughter therapy as a technique based on laughter and its usefulness in areas such as cardiovascular health, stress and cancer through A brief review of their literature.
\end{abstract}

Key words: Laugh, Laughter Therapy, Health.

\section{Introducción}

La risa es una manifestación de alegría, es un fenómeno biológico que produce cambios transitorios en la expresión facial, ${ }^{1}$ es una peculiaridad de la raza humana en respuesta a ciertos estímulos. ${ }^{2}$ Además, la risa tiene el poder de descargar sentimientos y emociones, facilitando respuestas fisiológicas, psicológicas y espirituales. ${ }^{3}$ Por otro lado y de acuerdo a lo expresado por Mora-Ripoll, ${ }^{4}$ la idea de que la risa está asociada a determinados beneficios de salud no es nueva; en las últimas décadas se han difundido diversas terapias que usan la risa y el humor con supuestos beneficios a nivel físico, emocional, social y espiritual. Desde la antigüedad el valor terapéutico de la risa franca y cordial es reconocida por sabios, filósofos y médicos; reír es bueno para la salud del cuerpo y del espíritu. ${ }^{5}$ Es así que, en el contexto clínico, muchos estudios médicos muestran que la risa tiene beneficios físicos y mentales, ${ }^{6}$ por lo que ésta puede utilizarse como intervención preventiva o como una alternativa a otras estrategias terapéuticas establecidas ${ }^{4}$ y en una variedad de contextos diferentes incluyendo: entrenamiento profesional, tratamiento del cáncer, agencias de salud mental y muchas otras dentro del campo médico ${ }^{7}$.

\section{La risa en el ámbito de la salud}

El Dr. William Evans al referirse a la risa expone: " $\mathrm{Si}$ usted tiene un dolor en su cuerpo y se pellizca en otro lugar, no sentirá tanto el primer dolor. La risa es como pellizcar la mente en un área y distraerla del dolor en otra". ${ }^{8}$

De acuerdo con Mora-Ripoll y García, ${ }^{9}$ la risa es una expresión de alegría, bienestar y descarga emocional, que permite la manifestación de diferentes estados de ánimo y emociones, constituyéndose en un componente esencial de comunicación del individuo. ${ }^{10}$ Libera tensiones y relaja el organismo, ${ }^{11}$ es capaz de reducir el estrés y la ansiedad, mejorando la calidad de vida y consecuentemente la salud física de las personas. ${ }^{12}$

La risa es un instrumento poderoso de curación que fortalece el sistema inmunológico del cuerpo, liberando endorfinas o llamadas también hormonas de la felicidad y adrenalina. ${ }^{13}$ Además activa la química de la voluntad de vivir, amplificando la capacidad para luchar contra la enfermedad, convirtiéndola de esta manera en una herramienta eficaz de autocuidado. ${ }^{14}$ Por otro lado y de acuerdo a lo expuesto en el artículo El valor terapéutico de la risa en medicina, la risa puede producir cambios fisiológicos sobre los sistemas musculo esquelético y cardiovascular; 
mejorar el estado anímico y consecuentemente desarrollar competencias sociales; incremento de catecolaminas afectando algunas funciones de manera positiva como el aumento de memoria, creatividad, respuesta interpersonal y otros neurotransmisores como la serotonina y dopamina, que tienen efectos antidepresivos y ansiolíticos $;{ }^{9}$ por lo que es considerada el medicamento más barato para prevenir muchas enfermedades y luchar contra ellas. ${ }^{15}$

La risa es algo personal e inherente a cada individuo; sin embargo se han establecido algunos tipos dependiendo de diferentes parámetros y campos de estudio; así desde el punto de vista médico y terapéutico existen cinco grandes grupos: 1) risa genuina o espontánea, desencadenada por diferentes estímulos y emociones positivas como la felicidad, regocijo, alegría entre otras; 2) risa auto inducida o simulada, producida a voluntad sin ninguna razón específica, por lo que no es provocada por el humor o por algo divertido, ni por emociones positivas; 3 ) risa estimulada, sucede como consecuencia de alguna acción o estímulo externo (p. ej. las cosquillas); 4) risa inducida, producida por la acción de diferentes fármacos o sustancias psicotrópicas (p. ej. Alcohol, cannabis, entre otras); y, 5) risa patológica, asociada a lesiones del sistema nervioso central como consecuencia de enfermedades neurológicas transitorias o persistentes (p. ej. Epilepsia gelástica, tumores, parálisis seudobulbar, entre otras), es producida sin ningún motivo aparente, ${ }^{16}$ así como también en enfermedades psiquiátricas como la esquizofrenia, demencia, entre otras. Este tipo de risa no se encuentra vinculada a cambios emocionales. ${ }^{9,17}$ La tabla 1 muestra un resumen de los tipos de risa.

Tabla 1. La risa desde el punto de vista médico y terapéutico

\begin{tabular}{|c|c|}
\hline Tipos de risa & Características \\
\hline Risa genuina & $\begin{array}{l}\text { Desencadenada por diferentes estímulos positivos. } \\
\text { Originada por emociones positivas. }\end{array}$ \\
\hline Risa auto inducida & $\begin{array}{l}\text { Producida sin una razón específica } \\
\text { No es provocada por emociones positivas, humor o } \\
\text { por situaciones divertidas. }\end{array}$ \\
\hline Risa estimulada & Desencadenada por acciones o estímulos externos. \\
\hline Risa inducida & $\begin{array}{l}\text { Producida por diferentes fármacos o sustancias psi- } \\
\text { cotrópicas. }\end{array}$ \\
\hline Risa patológica & $\begin{array}{l}\text { Consecuencia de enfermedades neurológicas tran- } \\
\text { sitorias o persistentes. } \\
\text { Resultado de enfermedades psiquiátricas }\end{array}$ \\
\hline
\end{tabular}

\section{Características}

Dentro del área médica, la risa es una reacción psicofisiológica que se caracteriza externamente por:

1) Contracciones enérgicas del diafragma, acompañadas de vocalizaciones silábicas que se repiten y tienen resonancia de la faringe, velo del paladar y otras cavidades fonatorias;

2) Expresión facial determinada con el movimiento de hasta 50 músculos del rostro, principalmente de aquellos que se encuentran alrededor de la boca, y puede ir acompañada de secreción lagrimal;

3) Movimientos de otros grupos musculares corporales como los de la pared abdominal, cabeza, cuello, espalda, brazos, piernas, entre otros;
4) Una serie de procesos neurofisiológicos asociados a cambios respiratorios y cardiovasculares, activación del sistema dopaminérgico y otros circuitos neuroendocrinos e inmunológicos. Internamente, la risa se acompaña de una sensación subjetiva reconocible (hilaridad). ${ }^{3}$

De acuerdo con Betés de Toro, ${ }^{18}$ además de las citadas anteriormente, la risa se caracteriza por:

1) Generar placer, pues contrae y afloja los músculos, lo que produce una sensación de relajación;

2) Es contagiosa;

3) Descarga de tensión, ya que al igual que el llanto produce un efecto relajante;

4) Provoca bloqueo de actividad mental, dado que durante la risa, disminuye la capacidad de pensar y de realizar movimientos;

5) Se potencia si no hay escape;

6) Es un mecanismo de defensa ante situaciones conflictivas.

Por lo tanto, los efectos de la risa en el organismo tienen resultados positivos tanto a nivel Psicológico como Fisiológico.

\section{Risoterapia}

La risoterapia es una técnica terapéutica que se encuentra fundamentada en los beneficios que proporciona la risa sobre la salud, calidad de vida y bienestar general para cualquier persona; contribuye a inmunizar contra enfermedades como la depresión, libera la tensión acumulada en el cuerpo, libera linfocitos $\mathrm{K}$, contribuye a fortalecer el sistema inmunitario ${ }^{19}$ y es un buen remedio anti estrés. ${ }^{20}$ Este es un enfoque diferente a los tratamientos médicos generales; se centra en la posición de que tanto el cuerpo como la mente pueden estar sanos sólo cuando se cambian los aspectos psicológicos del cerebro más allá del tratamiento físico, ${ }^{21}$ por lo que es considerada una terapia preventiva, terapéutica complementaria, alternativa a otros tratamientos establecidos ${ }^{22}$ y no invasiva, cuyo uso se ha extendido rápidamente, debido a la variedad de efectos positivos sobre la ansiedad, depresión, tensión, ira e incluso en el tratamiento de pacientes con cáncer, porque mejora la calidad de vida, la resiliencia, la inmunidad, ansiedad, depresión y estrés. ${ }^{23}$

Para comprender los beneficios que la risoterapia aporta al individuo es importante conocer la neurofisiología de la risa y cómo esta actúa en el organismo. Según Rojo de la Torre ${ }^{24}$ en el cerebro la risa reside en la zona más humana, en donde se encuentra la creatividad, la capacidad para pensar en el futuro y lo moral; se ubica en la parte dorsal de la unión del mesencéfalo con la protuberancia, en el seno de la sustancia gris periacueductal y la formación reticular, que mantiene conexiones con el cerebelo, hipotálamo, tálamo, ganglios basales, y lóbulos temporal y frontal. Este centro gelástico mesencéfalo pontino activará sincrónicamente los núcleos de los pares craneales que inervan los músculos implicados (expresión facial, vocalizaciones, espiración 
clónica) y los que determinan los fenómenos vegetativos asociados. El centro gelástico mesencéfalo se encuentra sujeto a tres sistemas: el inhibidor, el excitador y el modulador. ${ }^{1}$

Según lo expuesto por Parvizi et al., ${ }^{25}$ la risa se activa por estímulos percibidos dentro de un contexto cognitivosocial, detectados por sitios de inducción localizados en el telencéfalo, que incluyen la corteza pre frontal, ventromedial, la corteza cingulada anterior, la amígdala extendida y el estriado ventral; a su vez, los sitios de inducción activados operan en sitios efectores como las cortezas motoras, el hipotálamo, la sustancia gris periacueductal, los núcleos de los nervios craneales y las regiones pre motoras que interconectan el funcionamiento de estas estructuras. Además, durante la risa se ha detectado que las mujeres activan la corteza pre frontal izquierda más que los hombres, existiendo una activación máxima de las regiones mesolímbicas, que incluyen el núcleo accumbens, lo que implica una mayor respuesta de la red de recompensas. ${ }^{26}$ En la figura 1 se esquematiza las áreas encefálicas y circuitos implicados en la risa

Fig. 1. Esquema de las áreas encefálicas y circuitos implicados en la risa

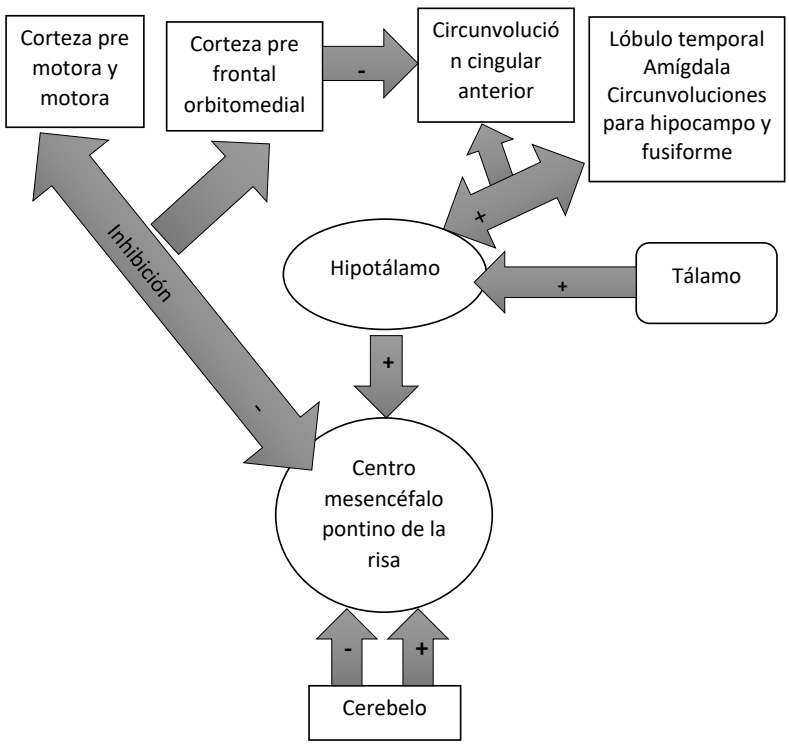

Adaptado de Arias, M. (2011)

\subsection{Sistema Cardiovascular}

Según Hoffman, ${ }^{7}$ las emociones negativas pueden contribuir a que exista mayor riesgo en enfermedades cardiovasculares. Muchos efectos negativos a dichas emociones van desde muerte cardiaca súbita hasta aturdimiento miocárdico temporal. En investigaciones realizadas por Miller y Fry, se determinó que la risa alegre causa efectos favorables a la biología endotelial y a la vasoconstricción. Se llegó a esta conclusión luego de realizar una investigación en un grupo de voluntarios, asignados a dos situaciones diferentes. Uno de los grupos pasó tiempo viendo una comedia popular de televisión mientras que el otro grupo vio una película que promovía el estrés mental; de las 160 mediciones recogidas se observó una reducción del $35 \%$ en vasodilatación de las personas del segundo grupo, en contraste con un aumento del $22 \%$ en vasodilatación de los voluntarios del primer grupo; llegando a la conclusión de que la risa y el humor promueven la salud vascular, pues la glándula pituitaria libera endorfinas que activan los receptores opiáceos en el endotelio vascular, responsables de la producción de óxido de nitrógeno, que a su vez favorece a los procesos celulares cardioprotectores que promueven la vasodilatación y reducen la inflamación vascular. ${ }^{27}$

\subsection{Estrés}

Uno de los métodos alternativos para la reducción del estrés es reír y estar en ambientes agradables. ${ }^{21}$ La risa es una sensación positiva que disminuye las hormonas que producen el estrés $^{28}$ como la epinefrina y el cortisol, ${ }^{29}$ eliminando los efectos que la misma provoca en el organismo, ${ }^{28}$ ayudando al individuo a lidiar con este, a reducir la ansiedad, promover la relajación, ${ }^{15}$ mejorar el autoestima y aumentar la activación del sistema de recompensa dopaminérgico mesolímbico. ${ }^{29}$

\subsection{Cáncer}

La risa es utilizada como terapia en pacientes que padecen de cáncer, cumpliendo una función psicológica, anestésica e inmunológica, dado que un buen estado de ánimo aumenta el conteo de células $\mathrm{T}$, así como la generación de células asesinas naturales que combaten enfermedades ${ }^{30}$ llamadas linfocitos que participan en la actividad citolítica contra las células tumorales y las células B, esenciales en la respuesta inmune. ${ }^{31}$ Cabe resaltar que muchos de los pacientes supervivientes de cáncer y los que han recurrido a la risa en sus recuperaciones han dado crédito a los aspectos positivos de ésta en la reducción del estrés y en la mejora de la actividad de las células asesinas naturales; uno de estos supervivientes Scott Burton dijo:

"Reacciones como la ira, depresión, negación, se llevaron un pedacito de mí con ellos; cada uno me hacía sentir menos humano. Sin embargo, la risa me hizo sentir más abierto a las ideas, más atractivo a los demás, e incluso un poco más fuerte interiormente. Me demostró que aunque mi cuerpo estaba devastado y mi espíritu desafiado, seguía siendo un ser humano". 32

\section{Conclusión}

La risoterapia dentro del contexto de la salud es considerada como un procedimiento alternativo, complementario y preventivo a otros tratamientos, que proporciona al organismo beneficios a nivel fisiológico, psicológico y espiritual. La risoterapia es una estrategia positiva que tiene influencia sobre la salud de las personas mejorando la calidad de vida de los pacientes.

Por lo expuesto anteriormente se concluye que la risoterapia debe ser incorporada en el ámbito de la salud como un medio útil y saludable en el tratamiento de enfermedades. 


\section{Referencias Bibliográficas}

1. Arias M. Neurología de la risa y del humor : risa y llanto patológicos. Revista de Neurología. 2011;53(7):415-421.

2. Elliot - Binns CP. Laughter and medicine. Journal of the Royal College of General Practitioners. 1985;(August):364-365.

3. Mora-Ripoll R. Medicina y Terapia de la Risa. Desclée De Brouwer, editor. Urduliz; 2010.

4. Mora-Ripoll R. Laughter techniques for therapeutic use in 28 . medicine. 2013;p. 1-7.

5. Sanz Ortiz JS. El humor como valor terapéutico. Elvieser. 2002;119(19):734-737.

6. Recker N. Laughter Is Really Good Medicine. 2007;.

7. Hoffmann MG. Benefits of Humor and Laughter : A Brief Introduction for Rehabilitation Counselors. 2013;.

8. Trent B. Ottawa lodges add humour to armamentarium in fight against cancer. Can Med asoc. 1990;142(2).

9. Mora-Ripoll R, García MC. El valor terapéutico de la risa en medicina. 2008;131(18):694-698.

10. Jaimes J, Claro A, Perea S, Jaimes E. La risa, un 31 . complemento esencial en la recuperación del paciente. $2011 ;(28)$.

11. Lotito Catino F. Gestionando con humor : un paso más hacia un liderazgo de excelencia. 2015;1(1):1-14.

12. Carbelo B, Jáuregui E. Emociones positivas: humor positivo. Papeles del Psicólogo. 2006;27:18-30.

13. Oblitas LA. PSICOLOGÍA DE LA SALUD : Una ciencia del bienestar y la felicidad. 2008;16(1).

14. Rippenger F C. The Connection between Laughter , Humor, and Good Health;.

15. Ghodsbin F, Ahmadi ZS, Jahanbin I, Farkhondeh S. O riginal A rticle The Effects of Laughter Therapy on General Health of Elderly People Referring to Jahandidegan Community Center in Shiraz ,. IJCBNM. 2015;3(1):3138.

16. Castellví E. El Taller de la risa. Alba, editor; 2011.

17. Mora-Ripoll R. The therapeutic value of laughter in medicine.pdf. Alternative Therapies. 2010;16:56-64.

18. Betés de Toro M. El humor como actitud ante la vida. HASER Revista Internacional de Filosofía Aplicada. 2011;2:67-93.

19. CEP de Villamartín. Curso : Risoterapia y Educación Emocional;.

20. Bernard L. La integración de técnicas de motivación y control de estrés, como medio didáctico, en la enseñanza de la expresión oral ( lengua extranjera ) a nivel superior . 2010;

21. Yim J. Therapeutic Benefits of Laughter in Mental Health : A Theoretical Review. 2016;p. 243-249.

22. Plaza Carmona M, Martínez González L. La risoterapia como complemento a otras terapias médicas. Revista enfermería Castilla y León. 2015;7:73-79.

23. Kim SH, Kim YH, Kim HJ. Laughter and Stress Relief in Cancer Patients : A Pilot Study. Hindawi Publishing Corporation. 2015;2015.

24. Rojo de la Torre S. Risoterapia y Bienestar; 2016.
25. Parvizi J, Anderson SW, Martin CO, Damasio H, Damasio AR. Pathological laughter and crying A link to the cerebellum. 2001;p. 1708-1719.

6. Nasr SJ. No laughing matter: Laughter is good psychiatric medicine treating mood disorders and. Current Psychiatry. 2013; (August).

27. Miller M, Fry W. The Effect of Mirthful Laughter on the Human Cardiovascular System. Med Hypotheses. 2010;73(5):1-7.

28. Farifteh S, Mohammadi-aria A, Kiamanesh A, Mofid B. The Impact of Laughter Yoga on the Stress of Cancer Patients before Chemotherapy. Autumn. 2014;7:179-183.

29. Savage BM, Lujan HL, Thipparthi RR, Dicarlo SE. Humor, laughter, learning, and health ! A brief review. The American Physiological Society. 2017;22:341-347.

30. Christian R, Ramos J, Susanibar C, Balarezo G. Risoterapia : Un nuevo campo para los profesionales de la salud Laugh Therapy : A new field for healthcare professionals. Revista de la sociedad peruana de la medicina interna. 2004;17(2):7-11.

1. Story Pattillo CG, Itano J. Laughter is the Best Medicine. AJN. 2001;(April):40-43.

32. Strean WB. Commentary Laughter prescription. Canadian Family Physician. 2009;55:965-967.

Recibido: 30 de octubre de 2017

Aceptado: 25 de noviembre de 2017 